\title{
EURRECA systematic review: how robust are biomarkers of zinc status?
}

\author{
N. M. Lowe ${ }^{1}$, K. Fekete ${ }^{2}$ and T. Decsi ${ }^{2}$ \\ ${ }^{1}$ Centre for Applied Sport and Exercise Sciences, University of Central Lancashire, Preston PR4 4AF, UK \\ and ${ }^{2}$ Department of Paediatrics, University of Pécs, Pécs, Hungary
}

The current recommendations for dietary $\mathrm{Zn}$ intake in adults range from $7 \mathrm{mg} / \mathrm{d}$ to $11 \mathrm{mg} / \mathrm{d}$ internationally ${ }^{(1)}$. This broad range reflects in part the difficulties and uncertainties associated with estimating $\mathrm{Zn}$ requirement for optimal health, which depends on a reliable indicator of status. One of the aims of the EURRECA Network of Excellence is to work towards aligning micronutrient intakes across Europe ${ }^{(2)}$. This process involved undertaking a systematic review of the relationship between dietary intakes of selected priority micronutrients and biomarkers of status in order to answer the primary question: which biomarkers of $\mathrm{Zn}$ status appropriately reflect change in status of $\mathrm{Zn}$ over durations of $\geq 2$ weeks. Secondary analysis was undertaken to explore the more detailed questions including the impact of age and ethnicity on the reliability of the biomarker. Medline, Embase and the Cochrane Library CENTRAL database were searched from inception to October 2007. Both supplementation and depletion studies were included.

Plasma or serum Zn concentration $(\mu \mathrm{mol} / \mathrm{l})$ was the most-frequently-reported biomarker of Zn status (fifty studies with 1454 participants). Primary analysis revealed that it responded to dietary manipulation across a range of $\mathrm{Zn}$ intakes from $<1 \mathrm{mg} / \mathrm{d}$ to $150 \mathrm{mg} / \mathrm{d}$ in healthy individuals (pooled effect 2.88 (95\% CI 2.24, 3.51)). Analysis of three studies with a total of ninety-three participants revealed that hair Zn concentration (ppm) was significantly elevated following supplementation (pooled effect 13.2 (95\% CI 11.9, 14.6)). Urinary $\mathrm{Zn}$ excretion $(\mu \mathrm{mol} / \mathrm{mmol}$ creatinine) also responded to $\mathrm{Zn}$ supplementation (five studies with 373 participants; pooled effect 0.31 (95\% CI $0.20,0.43)$ ). For other biomarkers, including platelet, polymorphonuclear cell and erythrocyte $\mathrm{Zn}$ concentrations and alkaline phosphatase activity, there were insufficient data to reach a definitive conclusion about their efficacy as biomarkers of $\mathrm{Zn}$ status.

The present review confirmed the eminence view that plasma and serum $\mathrm{Zn}$ concentration is a useful biomarker of $\mathrm{Zn}$ status in healthy individuals, responding to both $\mathrm{Zn}$ supplementation and depletion. The review highlighted a need for further research on a range of potentially-useful biomarkers including enzymes and other Zn-binding proteins for which there were insufficient data and for studies of vulnerable groups, such as infants and immigrant populations in Europe.

1. Geissler C \& Powers H (2005) Human Nutrition, 11th ed. London: Elsevier Churchill Livingstone.

2. Ashwell M, Lambert JP, Alles MS et al. (2008) Eur J Nutr 47, Suppl. 1, 2-16. 